\title{
MEASUREMENT AND INTERPRETATION OF THE ABSOLUTE INFRARED INTENSITIES OF ACETYLENE: FUNDAMENTALS AND COMBINATION BANDS
}

\author{
Th. KOOPS, W. M. A. SMIT and T. VISSER \\ Analytical Chemistry Laboratory, University of Utrecht, Croesestraat 77A 3522 AD \\ Utrecht (The Netherlands)
}

(Received 30 June 1983)

\begin{abstract}
The absolute gas-phase IR intensities of $\mathrm{C}_{2} \mathrm{H}_{2}$ and $\mathrm{C}_{2} \mathrm{D}_{2}$ have been measured by the Wilson-Wells-Penner-Weber method, using nitrogen as a broadening gas at a pressure of $60 \mathrm{~atm}$. The intensities of the fundamentals and the stronger combination bands have been determined. The fundamental intensities have been interpreted in terms of dipole moment derivatives, bond-charge parameters and atomic polar tensors. A comparison of the experimental parameters with corresponding ab initio calculated values is given. The sign and magnitude of some higher order terms in the dipole moment function of acetylene were obtained from an analysis based upon the experimental intensities of fundamentals and combination bands and the additional use of ab initio LMO bond moment calculations.
\end{abstract}

\section{INTRODUCTION}

Several authors have published the absolute IR intensities of $\mathrm{C}_{2} \mathrm{H}_{2}[1-6]$, and its deuterated derivatives $\mathrm{C}_{2} \mathrm{HD}[2,6]$ and $\mathrm{C}_{2} \mathrm{D}_{2}[2,4-6]$. The reported intensity values differ considerably in some cases, especially with respect to the $\nu_{s}$ bending mode. The lack of internal consistency of the measurements is clearly displayed by the $F$-sum rule results, which are unsatisfactory for all measurements, with the exception of the recent data of Kim and King [6]. In all cases the $F$-sum value for the $\nu_{5}$ bending mode of $\mathrm{C}_{2} \mathrm{H}_{2}$ appeared to be larger than the corresponding value for $\mathrm{C}_{2} \mathrm{D}_{2}$ suggesting that for this large amplitude mode the linear dipole approximation might not be valid, leading to a breakdown of the sum rule. However, recent $a b$ initio calculations performed in this laboratory [7] indicate that higher order dipole moment derivatives with respect to the $S_{5}$ bending coordinate scarcely contribute to the dipole moment function of acetylene, inferring the opposite conclusion. In view of these contradictory facts it seems worthwhile to re-establish accurately the absolute intensities of the fundamental modes of $\mathrm{C}_{2} \mathrm{H}_{2}$ and $\mathrm{C}_{2} \mathrm{D}_{2}$ in order to see whether the results of $\mathrm{Kim}$ and $\mathrm{King}$ [6] could be confirmed or not. Additionally, measurement of the intensities of the stronger combination 
bands may give some insight in the higher order contributions to the acetylene dipole moment function. The results of such measurements are now reported and discussea.

\section{EXPERIMENTAL}

The intensity measurements were carried out on a Perkin-Elmer model 180 spectrophotometer equipped with a data control interface and a digital magnetic recorder (PE 109). The instrument was flushed with dry and $\mathrm{CO}_{2}$ free air. A stainless steel absorption cell with KRS-5 windows and an effective pathlength of $5.15 \mathrm{~cm}$ was used. Sample pressures ranged from 0.0 to $95 \mathrm{~cm} \mathrm{Hg}$ for $\mathrm{C}_{2} \mathrm{H}_{2}$ and from 0.0 to $16 \mathrm{~cm} \mathrm{Hg}$ for $\mathrm{C}_{2} \mathrm{D}_{2}$. In order to achieve good accuracy for sample pressures lower than $2.5 \mathrm{~cm} \mathrm{Hg}$, carefully premixed samples of acetylene in nitrogen were used. The experimental intensities were measured accorcing to the Wilson-Wells-Penner-Weber method [ 8 , 91. The samples were pressurized by adding $60 \mathrm{~atm}$ of pure nitrogen. At this pressure and the applied spectral slit width (resolution better than $1.1 \mathrm{~cm}^{-1}$ ) the vibration-rotation lines were sufficiently broadened to give linear Beer's law plots as shown in Figs. $1-3$. The spectra were sampled with an interval of $1 \mathrm{~cm}^{-1}$ and numerically integrated. The $\mathrm{C}_{2} \mathrm{H}_{2}$ sample was obtained from Hoekloos (Amsterdam, The Netherlands) and contained about 3\% acetone as the only impurity. Aiter fractional distillation the acetone percentage was lowered to $0.2 \%$ as shown by the absolute intensity of the CO stretching mode [10]. All sample pressure measurements were corrected for this value. The following integration intervals were used for $\mathrm{C}_{2} \mathrm{H}_{2}: \nu_{1}+\nu_{5}, 4170-3980$; $\nu_{2}+2 \nu_{4}+\nu_{5} / \nu_{3}+\nu_{4}, 3970-3750 ; v_{3}, 3500-3100 ; \nu_{2}+v_{5}, 2780-2500 ; v_{4}$ $+\nu_{5}, 1500-1200$ and $\nu_{5}, 850-550 \mathrm{~cm}^{-1}$. The integration interval of $\nu_{5}$ also includes the $\nu_{7}$ fundarnental of the acetone impurity. Hence a correction was made based upon the $\nu_{7}$ intensity reported in ref. 10 for acetone. Similarly the intensity of the combination band $\nu_{4}+\nu_{5}$ was corrected for the intensiities of the $\nu_{15}, \nu_{4}, \nu_{21}, \nu_{16}$ and $\nu_{5}$ fundamentals of acetone. The separation of $\nu_{2}+2 \nu_{4}+\nu_{5}$ from $\nu_{3}+\nu_{4}$ was accomplished by assuming the intensity of the quarternary combination to be equally distributed on both sides of the peak maximum at $3898 \mathrm{~cm}^{-1}$ [11]. Thus, twice the area from $3898 \mathrm{~cm}^{-1}$ to the higher integration limit was subtracted from the total area. The remaining area was ascribed to the $\nu_{3}+\nu_{4}$ combination band. In order to account for the Fermi rescnance between $\nu_{3}$ and $\nu_{2}+\nu_{4}+\nu_{5}[12]$, the unperturbed intensity of the latter band was considered to be zero. Hence all intensity in the $\mathrm{CH}$ stretching region was assigned to the $\nu_{3}$ fundamental mode. The $\mathrm{C}_{2} \mathrm{D}_{2}$ sample was purchased from Merck, Sharp and Dohme, Canada Ltd. (purity 99 atom\% $\mathrm{D}$, implying the presence of about $2 \mathrm{~mol} \% \mathrm{C}_{2} \mathrm{HD}$ ) and was used without further purification. The stated purity was confirmed by careful comparison of our IR spectra with the intensity data of $\mathrm{C}_{2} \mathrm{HD}$ as given by $\mathrm{Kim}$ and King [6]. The sample pressure measurements as well as the intensity measurements were accordingly corrected. The integration intervals used for 

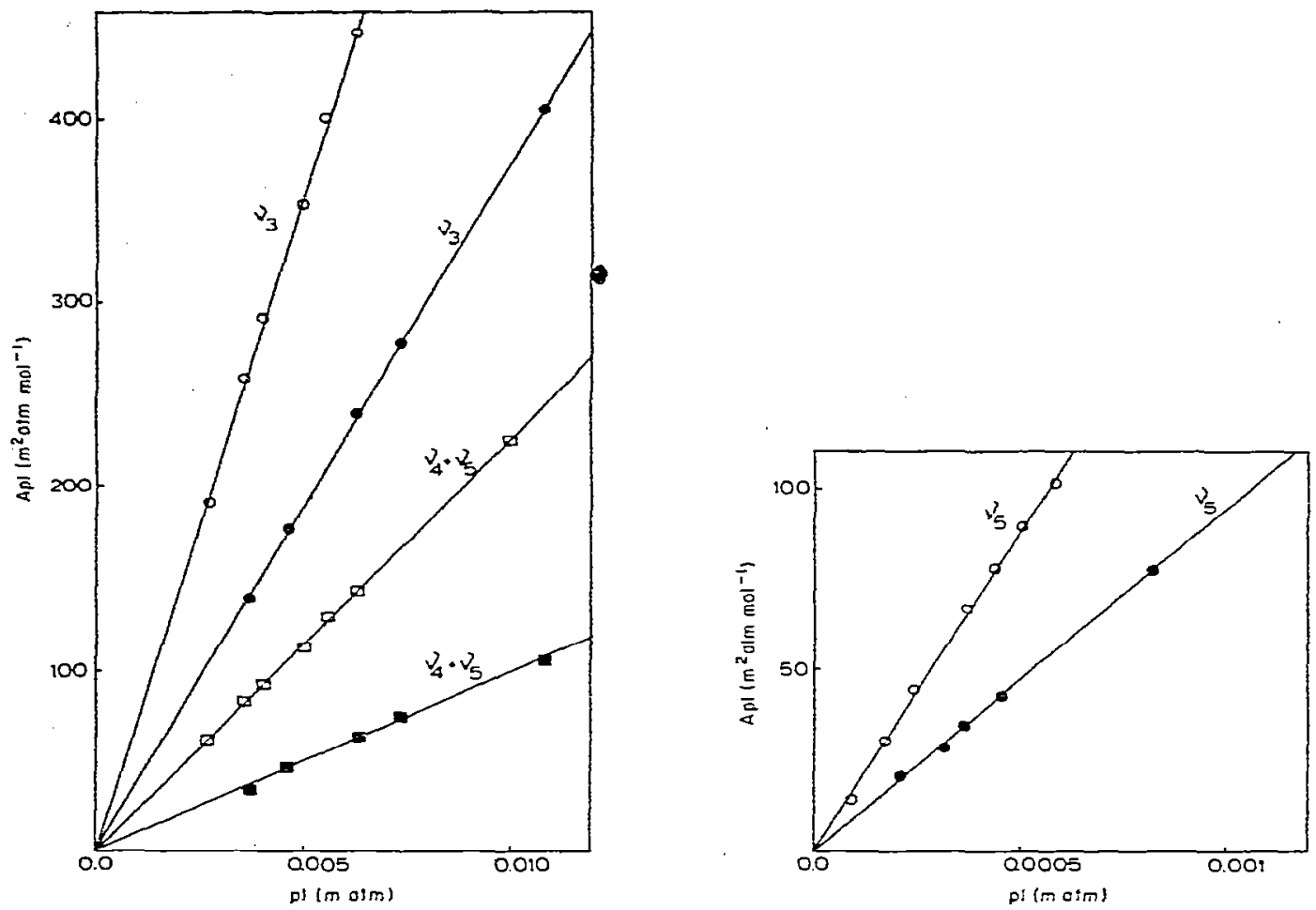

Fig. 1. Beer's law plots for some absorption bands of $C_{2} H_{2}$ and $C_{2} D_{2}: O_{2} \nu_{3} C_{2} H_{2} ; \bullet_{2} \nu_{3}$ $\mathrm{C}_{2} \mathrm{D}_{2} ; \square, v_{4}+v_{5} \mathrm{C}_{2} \mathrm{H}_{2} ; a, v_{4}+\nu_{5} \mathrm{C}_{2} \mathrm{D}_{2}$.

Fig. 2. Beer's law plots for the $v_{5}$ fundamentals of $\mathrm{C}_{2} \mathrm{H}_{2}$ and $\mathrm{C}_{2} \mathrm{D}_{2}: 0, v_{5} \mathrm{C}_{2} \mathrm{H}_{2} ; \bullet, v_{5} \mathrm{C}_{2} \mathrm{D}_{2}$.

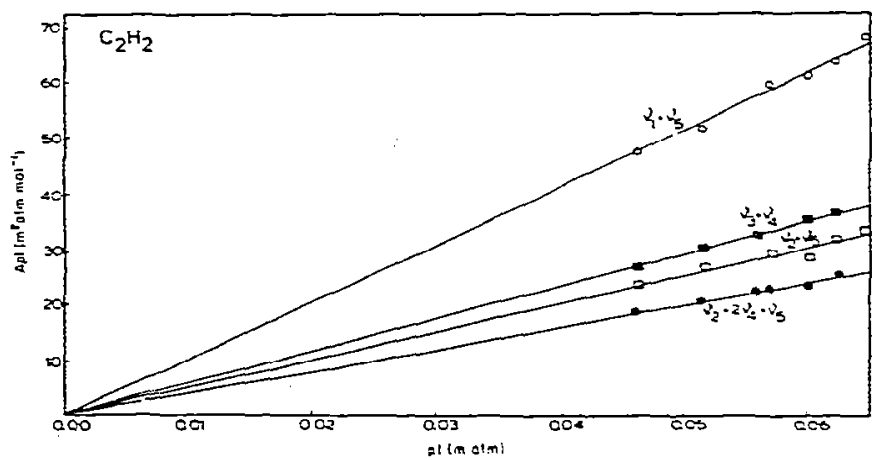

Fig. 3. Beer's law plots for some combination bands of $\mathrm{C}_{2} \mathrm{H}_{2}$. 
$\mathrm{C}_{2} \mathrm{D}_{2}$ were: $\nu_{3}, 2650-2250 ; \nu_{4}+\nu_{5}, 1150-1020$ and $\nu_{5}, 620-400 \mathrm{~cm}^{-1}$. Due to the limited amount of $C_{2} D_{2}$ on one hand and the weakness of the $\nu_{1}+\nu_{5}$, $\nu_{2}+\nu_{5}$ and $\nu_{3}+\nu_{4}$ combination bands on the other, their absolute intensities could not be determined.

The absolute intensities resulting from the Beer's law plots are given in Table 1, together with the corresponding literature values. The stated experimental errors in our intensity data are standard deviations resulting from a least-squares procedure in which errors in the observed band areas as well as in the sample pressures were taken into account [13].

It is rewarding to note that our new measurements are in excellent agreement with the values reported by Kim and King [6]. All intensities are within the stated error limits. Comparison of the recent values with our previous results [5] shows the latter being about $10 \%$ too high, with the exception of $\nu_{s}(d 2)$ for which a too low value was measured. Very likely, the high values of our previous measurements result from the fact that the samples were not premixed with nitrogen leading to errors in the sample pressure measurements. It is not clear, however, why such a low value was found for $v_{5}$ (d2). It is remarkable that the older intensity data for acetylene $[1-3]$ as listed in Table 1, despite rather poor experimental conditions (lower broadening pressure, less resolving power), closely correspond to the recent results, with the exception of the $\mathrm{C}_{2} \mathrm{D}_{2}$ intensities reported by Eggers et al. [2] .

The intemal consistency of the measurements may be checked by applying the $F$-sum rule $[18,19]$. The present $F$-suin values given in Table 2 for $\mathrm{C}_{2} \mathrm{H}_{2}$ and $\mathrm{C}_{2} \mathrm{D}_{2}$ are completely satisfactory; all differences are within the error limits. For comparison the $F$-sums are given for all reported acetylene intensities. The full agreement between our present results and those of Kim and King [6] again shows up, while the $F$-sum results for the measurements reported in refs. 2, 4 and 5 clearly reveal the presence of errors in the measuring procedures. As pointed out by. Jona et al. [20] an additional check for this molecule results from the relation $A_{i}\left(\mathrm{C}_{2} \mathrm{H}_{2}\right) / A_{i}\left(\mathrm{C}_{2} \mathrm{D}_{2}\right)=\left[L_{i}^{s}\left(\mathrm{C}_{2} \mathrm{H}_{2}\right) /\right.$ $\left.L_{i}^{s}\left(\mathrm{C}_{2} \mathrm{D}_{2}\right)\right]^{2}$. The present intensities and those of Kim and King [6] closely obey this relationship.

\section{RESULTS AND DISCUSSION}

\section{Dipole moment derivatives}

In the harmonic oscillator linear dipole approximation the relation between the absolute intensity $A_{i}$ and the derivative with respect to the normal coordinate $Q_{i}$ is given by

$A_{i}=N o \pi g_{i} / 3 c^{2}\left(\nu_{i} / \omega_{i}\right)\left(\partial \mu / \partial Q_{i}\right)^{2}$

where $N_{0}$ is Avogadro's number, $g_{i}$ the degeneracy of $Q_{i}, c$ is the velocity of light, $\nu_{i}$ and $\omega_{i}$ are the observed and harmonic frequencies respectively and $\mu$ is the molecular dipole moment. 

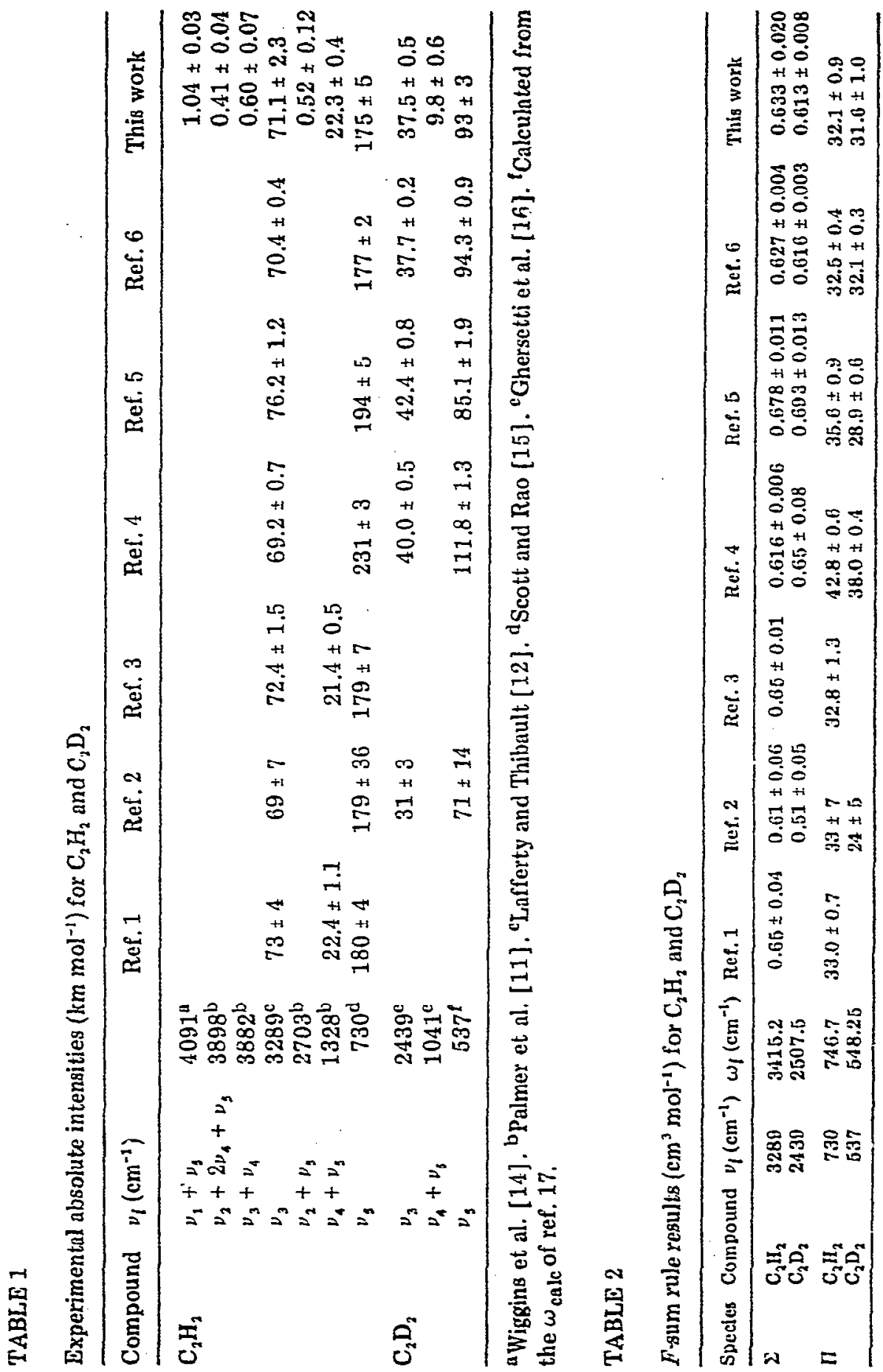
The dipoie moment derivatives with respect to the symmetry coordinates (dmd's) are obtained from the relation

$P_{S}=F_{Q} L^{-1}$

in which the elements of the $\mathbf{P}_{S}$ polar tensor are the dmd quantities, $\mathbf{P}_{Q}$ contains the derivatives with respect to normal coordinates and $\mathbf{L}^{-1}$ is defined by the linear transformation from symmetry to normal coordinates $\mathbf{Q}=\mathbf{L}^{-1} \mathbf{S}$. In Table 3 the symmetry coordinates are given in terms of internal coordinates the latter being defined in Fig. 4. The geometry parameters and harmonic force constants [17] are listed in the same Table.

The experimental $\partial \mu / \partial Q$ and $\partial \mu / \partial S$ valies are displayed in Table 4, while Table 5 contains a number of ab initio values for these quantities $[5,7,22-$ 24]. The corresponding dmd's for the ethynyl part of propyne, converted to the symmetry coordinates of Table 3 , are also given in Table 4 . In order to get comparable values, the propyne $\partial \mu_{x} / \partial S_{S_{x}}$ value has been corrected for the absclute rotational contribution given in ref, 21. As can be seen from Table 4 the experimental dmd values for $\mathrm{C}_{2} \mathrm{H}_{2}$ and $\mathrm{C}_{2} \mathrm{D}_{2}$ are in very good agreement with the corresponding propyre values, showing that the ethynyl part of propyne is very similar to acetylene. The $a b$ initio calculated dmd's listec in Table 5 are, as usual, all too high with the remarkable exception of the values given by Wiberg and Wendoloski [23] which are in excellent agreement with the present experimental values. The $a b$ initio results of Wiberg and Wencioloski were obtained by evaluating 4-31G results according to a Hybrid Orbital Rehybridization Model (HORM) which might be a promising concept for the calculation of molecular polar parameters. In the same paper they also report dmd values for methane which are in good agreement with recent experimental values from our laboratory [25]. However, the basis set

TABLE 3

Symmetry coordinates, geometry parameters and harmonic force constants for acetylene

\begin{tabular}{|c|c|}
\hline Species & Symmetry coordinates \\
\hline \multirow[t]{3}{*}{$\begin{array}{l}\Sigma_{\mathrm{g}} \\
\Sigma_{\mathbf{u}} \\
\Pi_{\mathbf{g}} \\
\Pi_{\mathbf{u}}\end{array}$} & $\begin{array}{ll}S_{1}=2^{-\frac{1}{2}}\left(\Delta r_{1}+\Delta r_{3}\right) & \\
S_{2}=\Delta r_{2} & \\
S_{3}=2^{\frac{1}{2}}\left(\Delta r_{1}-\Delta r_{3}\right) & \\
S_{4 x}=2^{-\frac{1}{2}}\left(\Delta \phi_{1 x}-\Delta \phi_{2 x}\right) A & S_{4 y}=2^{-\frac{1}{2}}\left(\Delta \phi_{1 y}-\Delta \phi_{2 y}\right) A \\
S_{5 x}=2^{-\frac{1}{2}}\left(\Delta \phi_{1 x}+\Delta \phi_{2 x}\right) \AA & S_{9 y}=2^{-\frac{1}{2}}\left(\Delta \phi_{1 y}+\Delta \phi_{2 y}\right) A\end{array}$ \\
\hline & $\begin{array}{l}\text { Geometry parameters } \\
r_{\mathrm{e}}(\mathrm{CH})=1.0605 \mathrm{~A} \quad r_{\mathrm{e}}(\mathrm{C} \equiv \mathrm{C})=1.2033 \mathrm{~A}\end{array}$ \\
\hline & $\begin{array}{ll}\text { Force constants } & \left(\mathrm{mdyn} \mathcal{A}^{-1}\right) \\
F_{11}=6.3510 & F_{33}=6.3890 \\
F_{12}=-0.1344 & F_{44}=0.1585 \\
F_{22}=16.3410 & F_{35}=0.3435\end{array}$ \\
\hline
\end{tabular}

astrey and Mills [17]. 


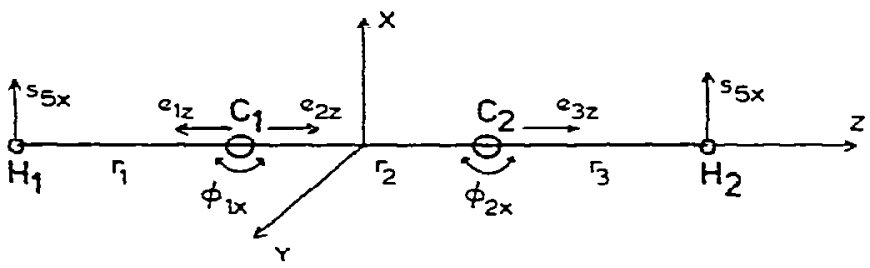

Fig. 4. Definition of internal coordinates and Cartesian axis system for acetylene.

\section{TABLE 4}

Experimental dipole moment derivative values for acetylene and the corresponding derivative values for propyne

\begin{tabular}{lrrrr}
\hline $\begin{array}{l}\text { Dipole moment } \\
\text { derivative }\end{array}$ & \multicolumn{1}{c}{$\mathrm{C}_{2} \mathrm{H}_{2}$} & \multicolumn{1}{c}{$\mathrm{C}_{2} \mathrm{D}_{2}$} & Average & Propyne $^{c}$ \\
\hline$\partial \mu_{z} / \partial Q_{3}\left(\mathrm{D} a m u^{-\frac{1}{2}} \mathrm{~A}^{-1}\right)$ & $-1.321 \pm 0.030$ & $-0.955 \pm 0.009$ & & \\
$\partial \mu_{x} / \partial Q_{5 x_{b}}$ & $1.455 \pm 0.029$ & $1.060 \pm 0.024$ & & \\
$\partial \mu_{x} / \partial Q_{5 x}$ & $1.439 \pm 0.030$ & $1.057 \pm 0.022$ & & \\
$\partial \mu_{z} / \partial S_{3}\left(\mathrm{D} \mathrm{A} \mathrm{A}^{-1}\right)$ & $-1.273 \pm 0.029$ & $-1.254 \pm 0.012$ & $-1.26 \pm \pm 0.016$ & $-1.232 \pm 0$ \\
$\partial \mu_{x} / \partial S_{5 x}$ & $1.488 \pm 0.030$ & $1.476 \pm 0.033$ & $1.482 \pm 0.022$ & $1.519 \pm 0$ \\
$\partial \mu_{x} / \partial S_{5 x}$ & $1.472 \pm 0.030$ & $1.472 \pm 0.030$ & &
\end{tabular}

a Obtained from the experimental intensity using the double harmonic approximation. ${ }^{b}$ Obtained from the anharmonic approximation, using the final signs $(-\rightarrow)$ for $\partial^{2} \mu{ }^{2} !$ $\partial Q_{1} \partial Q_{5 x}$ and $\partial^{2} \mu_{x} / \partial Q_{2} \partial Q_{5 x}$ (see text). ${ }^{c}$ Bode et al. [21]. The minus sign for $\partial \mu_{2} / \partial S_{3}$ results from the fact that propyne has been given a position within the axis system similar to acetylene (see Fig.4).

\section{TABLE 5}

Ab initio calculated dipole moment derivatives for acetylene $\left(D A^{-1}\right)$

\begin{tabular}{lrrrrr}
\hline $\begin{array}{l}\text { Dipole moment } \\
\text { derivative }\end{array}$ & Ref. 22 & Ref. 5 & Ref. 23 & Ref. 7 & Ref. 24 \\
\hline$\partial_{\mu_{z}} / \partial S_{3}$ & -1.365 & -1.540 & -1.228 & -1.544 & -1.418 \\
$\partial \mu_{x} / \partial S_{5 x}$ & 1.988 & 2.032 & 1.482 & 1.658 & 1.572 \\
\hline
\end{tabular}

sensitivity of the HORM results should be further analyzed in order to get more insight in the usefulness of this model. In the same paper Wiberg and Wendoloski propose a new method to analyse LMO results for the calculation of bond moments. This method certainly deserves further attention. In general, the $a b$ initio values of Table 5 are all in reasonable agreement with the experimental values, while the spread in the predicted values clearly shows the rather strong basis set dependency of the calculated quantities.

The absolute intensities for the binary combination bands given in Table 1 were reduced to the transition moment values listed in Table 6 , by using the following expression derived by Yao and Overend [26] 
TABLE 6

Experimental transition moment values for binary combination bands of $C_{2} H_{2}$ and $C_{2} D_{2}$ and values for the mechanical anhar noinicity term of eqn. (3). All values in $\mathrm{D} \times 10^{4}$

\begin{tabular}{llllc}
\hline Compound & $i$ & $j$ & $\left\langle v_{i}=0, v_{j}=0 \mid \mu_{\xi} i v_{i}=1, v_{j}=1\right\rangle^{\mathrm{a}}$ & $\begin{array}{c}\text { Mechanical } \\
\text { anharmonicity term }\end{array}$ \\
\hline $\mathrm{C}_{2} \mathrm{H}_{2}$ & 1 & 5 & $\pm 70.1 \pm 1.4$ & 0.00 \\
& 2 & 5 & $\pm 61 \pm 10$ & 0.00 \\
& 3 & 4 & $\pm 54 \pm 5$ & $70.8 \pm 1.4$ \\
$\mathrm{C}_{2} \mathrm{D}_{2}$ & 4 & 5 & $\pm 784 \pm 10$ & $222 \pm 5$ \\
\end{tabular}

$a_{\xi}=x, y$ or $z$.

$$
\begin{aligned}
& \Gamma_{v_{i}, v_{j}}^{v_{i}+v_{j}+1}=\frac{2 \pi^{3} N o}{3 h c}\left(\frac{2}{v_{i+1}}\right)\left(\frac{2}{v_{j+1}}\right) Z_{i} Z_{j}\left\langle v_{i}=0, v_{j}=0|\mu| v_{i}=1, v_{j}=1\right\rangle^{2} \\
& \quad \times\left(1-\mathrm{e}^{-\beta\left(\omega_{i}+\omega_{j}\right)}\right)=\frac{2 \pi^{3} N o}{3 h c} Z_{i} Z_{j}\left[\frac{\partial^{2} \mu}{\partial q_{i} \partial q_{j}}+\left[2\left(\frac{\partial \mu}{\partial q_{i}}\right) \frac{K_{i i j} \omega_{i}}{\omega_{j}\left(2 \omega_{i}+\omega_{j}\right)}\right.\right. \\
& \quad+2\left(\frac{\partial \mu}{\partial q_{j}}\right) \frac{K_{i j i} \omega_{j}}{\omega_{i}\left(2 \omega_{j}+\omega_{i}\right)}-\sum_{k \neq i, j} g_{5}\left(i j k j\left(\frac{\partial \mu}{\partial q_{k}}\right) \omega_{k}\left\{\omega_{k}^{2}-\left(\omega_{i}-\omega_{j}\right)^{2}\right\}\right]^{2} \\
& \quad \times\left(1-e^{-\beta\left(\omega_{i}+\omega_{j}\right)}\right)
\end{aligned}
$$

where $\Gamma$ denotes the combination band intensity in $\mathrm{m}^{2} \mathrm{~mol}^{-1}$, No is Avogadro's number, $h$ is Planck's constant, $c$ is the velocity of light, $Z_{i}$ is the vibrational partition function given by $Z_{i}=\left(1-e^{-\beta \omega_{i}}\right)^{-1}$, with $\beta=h c / k T$. The last part of eqn. (3) describes the different contributions to the combination band intensities. The quantity $\partial \mu / \partial q_{i}$ denotes the derivative of the molecular dipole moment with respect to the $i$ th dimensionless normal coordinate, the latter being defined by $q_{i}=\left(4 \pi^{2} c \omega_{i} / h\right)^{1 / 2} Q_{i} . K_{i i j}$ is the cubic force constant $\left(\mathrm{cm}^{-1}\right)$ on dimensionless normal coordinate basis. The explicit expression for $g_{5}(i j k)$, which depends on the cubic force constant $K_{i j k}$ and the pertaining harmonic frequencies, is as follows $g_{s}(i j k)=K_{i j k}\left[\left(\omega_{i}+\omega_{j}+\omega_{k}\right)\left(\omega_{i}-\omega_{j}-\omega_{k}\right)\left(\omega_{i}-\right.\right.$ $\left.\left.\omega_{j}+\omega_{k}\right)\left(\omega_{i}+\omega_{j}-\omega_{k}\right)\right]^{-1}$. In order to calculate the transition moments from eqn. (3) the experimental $A_{i}$ values were converted to $\Gamma_{i}$ values by use of the relation $\Gamma_{i}=A_{i} / \nu_{i}$, while the temperature of the samples during the measurements was determined to be $309 \mathrm{~K}$. As shown by eqn. (3), the electric dipole vibrational transition moment consists of an electric anharmonicity term, viz. the term containing the second derivative of the dipole moment, and three terms depending on the cubic force field, together representing the mechanical anharmonicity term. The mechanical anharmonicity terms are also given in Table 6 . The cubic force constants used in the calculations were taken from Domingo et al. [27], who transformed the cubic force field of Strey and Mills [17] to a dimensionless normal coordinate basis. Inspection 
of Table 6 reveals that, with the exception of $v_{3}+v_{4}$, the combination band intensities are dominated by the electrical anharmonicity term. From the values for the mechanical anharmonicity terms and the experimental transition moments as given in Table 6 , the $\partial^{2} \mu / \partial Q_{i} \partial Q_{j}$ quantities were calculated and collected in Table 7 . Two values for each second derivative are found, depending on the sign of the transition moment. The $\partial^{2} \mu / \partial Q_{i} \partial Q_{j}$ values were further converted to $\partial^{2} \mu / \partial S_{i} S_{j}$ quantities, which are given in the same Table. As can be seen from Table 7 , the calculated $\partial^{2} \mu_{x} / \partial S_{4} \partial S_{5 x}$ values for $\mathrm{C}_{2} \mathrm{H}_{2}$ and $\mathrm{C}_{2} \mathrm{D}_{2}$ are slightly different. These differences for the positive signs are however, not significant when taking into account the experimental errors in the measured $\nu_{4}+\nu_{5}$ intensities, as given in Table 1 . In order to decide which $\partial^{2} \mu / \partial Q_{i} Q_{j}$ values are the more preferable ones, we have used the $\mathrm{C}_{2} \mathrm{H}_{2}$ results to predict the intensities of the $\mathrm{C}_{2} \mathrm{D}_{2}$ combination bands. By use of the linear transformation $\mathbf{Q}=\mathbf{L}^{-1} \mathbf{S}$ the $\partial^{2} \mu / \partial S_{i} \partial S_{j}$ quantities were converted to $\left(\partial^{2} \mu / \partial Q_{i} \partial Q_{j}\right)_{C_{2} D_{2}}$ parameters, which were in turn converted to $\left(\partial^{2} \mu / \partial q_{i} \partial q_{j}\right)_{C_{2} D_{2}}$ values. From these values and the pertaining mechanical anharmonicities the absolute intensities for the binary combination bands of $C_{2} D_{2}$, as listed in Table 8 , were calculated. Since $\nu_{1}$ and $\nu_{2}$ belong to the same symmetry species, $\partial^{2} \mu_{x} / \partial Q_{1} \partial Q_{5 x}$ and $\partial^{2} \mu_{x} / \partial Q_{2} \partial Q_{5 x}$ are both functions of $\partial^{2} \mu_{x} / \partial S_{1} \partial S_{5 x}$ and $\partial^{2} \mu_{x} / \partial S_{2} \partial S_{5 x}$. This leads to four solutions for the $\partial^{2} \mu_{x} /$ $\partial S_{1} \partial S_{5_{x}}$ and $\partial^{2} \mu_{x} / \partial S_{2} \partial S_{5_{x}}$ parameters (see footnote ${ }^{b}$ Table 7 ).

Since the contribution of mechanical anharmonicity to the $\nu_{1}+\nu_{5}$ and $v_{2}$ $+v_{5}$ intensities is zero, the calculated intensities do not depend on the sign of the $\partial^{2} \mu / \partial S_{i} \partial S_{j}$ parameters, thus giving rise to only two different intensity values as given in Table 8 . Also two intensity values are found for the $v_{3}+\nu_{4}$ and $\nu_{4}+\nu_{5}$ combination bands of $\mathrm{C}_{2} \mathrm{D}_{2}$. At first glance this may look confusing, since the different $\partial^{2} \mu / \partial S_{i} \partial S_{j}$ values both for $\nu_{3}+\nu_{4}$ and $\nu_{4}+\nu_{5}$ lead to

\section{TABLE 7}

Second derivatives of the dipole moment with respect to normal-and symmetry coordinates

\begin{tabular}{|c|c|c|c|c|c|c|}
\hline \multirow[t]{2}{*}{ Compound } & \multirow[t]{2}{*}{$i$} & \multirow[t]{2}{*}{$j$} & \multicolumn{2}{|c|}{$\partial^{2} \mu / \partial Q_{i} \partial Q_{j}{ }^{2}\left(\mathrm{D} \mathrm{amu}^{-1} A^{-2}\right)$} & \multicolumn{2}{|c|}{$a^{2} \mu / \partial S_{i} \partial S_{j}^{b}\left(D \AA^{-2}\right)$} \\
\hline & & & I & II & III & IV \\
\hline $\mathrm{C}_{2} \mathrm{H}_{2}$ & $\begin{array}{l}1 \\
2 \\
3 \\
4\end{array}$ & $\begin{array}{l}5 \\
5 \\
4 \\
5\end{array}$ & $\begin{array}{l}+0.672 \pm 0.013 \\
+0.44 \pm 0.07 \\
-0.15 \pm 0.04 \\
+2.28 \pm 0.05\end{array}$ & $\begin{array}{l}-0.672 \pm 0.013 \\
-0.44 \pm 0.07 \\
-1.08 \pm 0.04 \\
-4.07 \pm 0.05\end{array}$ & $\begin{array}{l} \pm 1.20 \pm 0.08 \\
\pm 0.97 \pm 0.18 \\
-0.12 \pm 0.03 \\
+1.94 \pm 0.04\end{array}$ & $\begin{array}{l} \pm 0.20 \pm 0.08 \\
\mp 1.21 \pm 0.18 \\
-0.87 \pm 0.03 \\
-3.46 \pm 0.04\end{array}$ \\
\hline $\mathrm{C}_{2} \mathrm{D}_{2}$ & 4 & 5 & $+1.34 \pm 0.08$ & $-2.30 \pm 0.08$ & $+1.86 \pm 0.11$ & $-3.20 \pm 0.11$ \\
\hline
\end{tabular}

${ }^{a}$ Columns I and II belong to positive and negative transition moment values respectively. bThe first two values in column III belong to the $+\dot{T}$ and - - sign combinations for $\partial^{2} \mu_{x} / \partial Q_{1} \partial Q_{5 x}$ and $\partial^{2} \mu_{x} / \partial Q_{2} \partial Q_{5 x}$, while the corresponding values in column IV belong to the +- and $\rightarrow$ combinations. In all cases the signs of the $\partial^{2} \mu / \partial S_{i} \partial S_{j}$ parameters are identical to the signs of the corresponding $a^{2} \mu / \partial Q_{i} \partial Q_{j}$ quantities. 
TABLE 8

Predicted intensities ( $\mathrm{km} \mathrm{mol} \mathrm{m}^{-3}$ ) ior binary combination bands of $\mathrm{C}_{2} \mathrm{D}_{2}$

\begin{tabular}{|c|c|c|c|c|c|}
\hline \multirow[t]{2}{*}{ Species } & \multirow[t]{2}{*}{$i$} & \multirow[t]{2}{*}{$\dot{j}$} & \multirow{2}{*}{$\nu_{i}+\nu_{j}\left(\mathrm{~cm}^{-1}\right)$} & \multicolumn{2}{|l|}{$A \nu_{i}+\nu_{j}$} \\
\hline & & & & $\mathbf{I}$ & II \\
\hline$\Pi_{u}$ & $\begin{array}{l}1 \\
2 \\
3\end{array}$ & $\begin{array}{l}5 \\
5 \\
4\end{array}$ & $\begin{array}{l}3235^{a} \\
2298^{b} \\
2944^{a}\end{array}$ & $\begin{array}{l}0.007 \pm 0.015 \\
0.04 \pm 0.02 \\
0.17 \pm 0.03\end{array}$ & $\begin{array}{l}0.83 \pm 0.17 \\
0.37 \pm 0.07 \\
0.40 \pm 0.04\end{array}$ \\
\hline$\Sigma_{u}$ & 4 & 5 & $1041^{c}$ & $10.45 \pm 0.30$ & $11.88 \pm 0.34$ \\
\hline
\end{tabular}

sRef. $28 .{ }^{b}$ Ref. $29 .{ }^{c}$ Ref. 16.

the same intensity value in the case of $\mathrm{C}_{2} \mathrm{H}_{2}$. However, the electrical and mechanical anharmonicity terms transform differently under isotopic substitution, leading to different intensity values in the case of $\mathrm{C}_{2} \mathrm{D}_{2}$. As can be seen from Table 8, there are marked differences between the predicted intensities for each of the combination bands. Comparison of the experimental combination band intensities for $\mathrm{C}_{2} \mathrm{D}_{2}$ with values predicted from $\mathrm{C}_{2} \mathrm{H}_{2}$ would therefore allow selection of the more preferable $\partial^{2} \mu / \partial S_{i} \partial S_{j}$ values. Since only the absolute intensity of the $\nu_{4}+\nu_{5}$ combination band of $C_{2} D_{2}$ was determined, this criterion could only be used for $\partial^{2} \mu_{x} / \partial S_{4} \partial S_{5 x}$. The best agreement between the experimental and calculated intensity for this combination band is obtained with a positive $\operatorname{sign}$ for $\partial^{2} \mu_{x} / \partial S_{4} \partial S_{5 x}$ (see Tables 1 and 8 for experimental and predicted intensity values respectively). In order to get information about the magnitudes and signs of the $\partial^{2} \mu_{x} / \partial S_{1} \partial S_{S_{x}}$ and $\partial^{2} \mu_{x} / \partial S_{2} \partial S_{5 x}$ parameters other arguments have to be used, which will be deveioped in the following. As shown in Tables 7 and 8 , the predicted $\nu_{1}+$ $\nu_{5}(d 2)$ and $\nu_{2}+\nu_{5}(d 2)$ intensities stzungly depend on the sign combination used. The ++ and -- combinations lead to nearly zero intensities, while the +- and -+ combinations result in intensities which are of comparable strength with the corresponding $\mathrm{C}_{2} \mathrm{H}_{2}$ intensities. Careful inspection of the $\mathrm{C}_{2} \mathrm{D}_{2}$ spectra, obtained at the highest available sample pressures, shows weak features at the $\nu_{1}+\nu_{5}$ and $\nu_{2}+\nu_{5}$ positions, in favour of the predictions with the +- and -+ sign combinations. The ++ and -- sign combinations must therefore be rejected.

In order to determine which of the two remaining sign combinations is the correct one, use was made of ab initio LMO bond mornent calculations $((9,5$, $4,2 / 4,2)$ Gaussian wavefunction, Magnasco-Perico localization method; for further details see ref. 7). The results are given in Table 9 , which contains the bond moments for the equilibrium geometry as well as for two distorted configurations, obtained after displacements along $S_{2}$ and $S_{5_{x}}$ respectively. This Table reveals that upon distortion along $S_{z}$ the $\mathrm{CH}$ bond moments are diminished. Furthermore, a distortion along $S_{5 x}$ produces a moment (dipole direction $-\rightarrow+$ ) of $0.0818 \mathrm{D}$ in the positive $x$-axis direction, built up from a 


\section{TABLE 9}

Bond moments (D) from LMO calculations for acetylene at the equilibrium and at two distorted configurations

\begin{tabular}{|c|c|c|c|c|}
\hline Bond & $\begin{array}{l}\text { Equilibrium } \\
\mathrm{Hz}\end{array}$ & $\begin{array}{l}\Delta S_{2}=0.02 \mathrm{~A} \\
\mu_{z}\end{array}$ & $\begin{array}{l}\Delta S_{s x}=2 \sqrt{ } 2^{\circ} \\
\mu_{x}\end{array}$ & $\mu_{z}$ \\
\hline $\begin{array}{l}\mathrm{C}_{2}-\mathrm{C}_{2} \\
\mathrm{C}_{1}-\mathrm{H}_{1} \\
\mathrm{C}_{2}-\mathrm{H}_{2}\end{array}$ & $\begin{array}{r}0.0000 \\
-1.2857 \\
1.2857\end{array}$ & $\begin{array}{r}0.0000 \\
-0.8115 \\
0.8115\end{array}$ & $\begin{array}{r}0.1904 \\
-0.0543 \\
-0.0543\end{array}$ & $\begin{array}{r}0.0000 \\
-1.2181 \\
1.2181\end{array}$ \\
\hline
\end{tabular}

positive moment in the $\mathrm{C} \equiv \mathrm{C}$ bond due to rehybridization of the LMOs that form the triple bond, and a negative moment in each of the $\mathrm{CH}$ bonds (combined effects of displacement of the $H$ atom along the positive $x$-axis and the corresponding change in the moment of the $\mathrm{CH}$ LMO). The total dipole moment change of $0.0818 \mathrm{D}$ leads to a value for $\partial \mu_{x} / \partial S_{5 x}$ of $1.659 \mathrm{D} \AA^{-1}$, as reported earlier [7]. Here it is of interest to see that the total dipole moment change along $S_{5 x}$ consists of two opposite contributions. The negative contribution, which originates from the $\mathrm{CH}$ bonds, may be expected to decrease upon decreasing the $\mathrm{CH}$ bond moment. This is exactly what occurs upon a combined distortion along $S_{2}$ and $S_{5_{x}}$, leading to the conclusion that $\partial^{2} \mu_{x}$ l $\partial S_{2} \partial S_{5 x}$ is positive. Therefore, the final sign combination for $\partial^{2} \mu_{x} / \partial S_{1} \partial S_{5 x}$ and $\partial^{2} \mu_{x} / \partial S_{2} \partial S_{s_{x}}$ and also for the corresponding normal coordinate derivatives is - + .

The last derivative to be considered is $\partial^{2} \mu_{2} / \partial S_{3} \partial S_{4}$. This derivative has two possible magnitudes, both with negative sign. Unfortunately however, the predicted $\mathrm{C}_{2} \mathrm{D}_{2}$ combination band intensities differ too little to allow the selection of the correct value for this derivative from the weak $\nu_{3}+\nu_{4}$ absorption feature in the $\mathrm{C}_{2} \mathrm{D}_{2}$ spectra. The calculated magnitudes and signs for the discussed second order dipole moment derivatives make it possible to describe the absolute intensity of the $\nu_{5}$ fundamental in the anharmonic approximation. The second order parameters occurring in the dipole moment function for $\nu_{5}$ are $\partial^{2} \mu_{x} / \partial Q_{1} \partial Q_{5 x}$ and $\partial^{2} \mu_{x} / \partial Q_{2} \partial Q_{5 x}$. By use of the expression given by Yao and Overend [26] for the absolute intensity in the anharmonic approximation we calculated by an iterative procedure the $\partial \mu_{x} / \partial Q_{5_{x}}$ value that belongs to the second order approximation of the dipole moment function, starting from the $\partial \mu_{x} / \partial Q_{5 x}$ value obtained from a "double harmonic" interpretation of $A_{5}$. The calculated $\partial \mu_{x} / \partial Q_{5 x}$ (second order approximation) value is included in Table 4 and the corresponding $C_{2} D_{2}$ value, obtained by transforming the $\mathrm{C}_{2} \mathrm{H}_{2}$ value, is given in the same Table. Since no information is available about the higher order terms, contributing to the $\nu_{3}$ fundamental intensity, no "harmonic" $\partial \mu_{z} / \partial Q_{3}$ parameter could be calculated. 
Bond charge parameters and atomic polar tensors

The dipole moment derivatives with respect to internal coordinates can be written in terms of bond charge parameters (bcp's) [30, 31]

$\partial \mu / \partial R_{j}=\sum_{k} \sum_{\sigma}\left\{r_{k} \mathbf{e}_{k, \sigma}\left(\partial q_{k} / \partial R_{j}\right)+q_{k} \mathbf{e}_{k, \sigma}\left(\partial r_{k} / \partial R_{j}\right)+q_{k} r_{k}\left(\partial \mathbf{e}_{k, \sigma} / \partial R_{j}\right)\right\}$

where $q_{k}$ denotes the effective bond charge of the $k$ th bond, $r_{k}$ the bond length and $e_{k, \sigma}$ denotes the $\sigma$-component $(\sigma=x, y$ or $z$ ) of the bond unit vector. The bond unit vector $e_{k}$ has the same direction as the corresponding bond moment $\mu_{k}$, which points from the negatively charged end of the bond to the positively charged end. In the case of zero bond moments the direction of the bond unit vector may be chosen arbitrarily from one end of the bond to the other. The value of $\mathbf{e}_{k_{v} \sigma}$ is positive when the component vector points in the positive axis-direction.

The above equation is based upon the assumption that the molecular dipole moment is the vector sum of bond moments

$\mu=\sum_{k} \mu_{k}$

Writing the bond moment of the $k$ th bond as

$\mu_{k}=\sum_{\sigma} \mu_{k, \sigma}=\sum_{\sigma} q_{k} r_{k} \mathbf{e}_{k, \sigma} \quad(\sigma=x, y$ or $z)$

eqn. (4) is easily derived. The quantities $q_{k}$ and $\partial q_{k} / \partial R_{j}$ are the desired bcp's, $q_{k}$ being the bond charge and $\partial q_{k} / \partial R_{j}$ the bond charge reorganization parameters. The latter parameters represent the charge fluxes occurring upon deformations along the internal coordinates $R_{j}$. In the case of acetylene there are two different bond charge equations, which may be evaluated to be

$\partial \mu_{z} / \partial r_{1}=r_{1}\left(\partial q_{1} / \partial r_{1}\right) e_{1 z}+q_{1} e_{1 z}+r_{2}\left(\partial q_{2} / \partial r_{1}\right) e_{2 z}+r_{3}\left(\partial q_{3} / \partial r_{1}\right) e_{3 z}$

and

$\partial \mu_{x} / \partial \alpha_{1 x}=r_{1} q_{1}\left(\partial \mathrm{e}_{1 z} / \partial \alpha_{1 x}\right)=r_{1} q_{1} \mathbf{e}_{1 x}$

It follows from inspection of Table 3 that

$\partial \mu_{z} / \partial S_{3}=2^{1 / 2}\left(\partial \mu_{z} / \partial r_{1}\right)$ and $\partial \mu_{x} / \partial S_{5 x}=2^{1 / 2}\left(\partial \mu_{x} / \partial \alpha_{1 x}\right)$

Equations (7)-(9) lead to the expressions for the dmd's in terms of bcp's as given in Table 10. These expressions can be solved for $q_{\mathrm{cH}}$, the bond charge of the $\mathrm{CH}$ bond and the charge flux term $\left[\left(\partial q_{1} / \partial r_{1}\right)-\left(\partial q_{3} / \partial r_{1}\right)-\gamma\left(\partial q_{2} / \partial r_{1}\right)\right]$. The calculated values for $\mathrm{C}_{2} \mathrm{H}_{2}$ and $\mathrm{C}_{2} \mathrm{D}_{2}$ are given in Table 10. The indicated errors are propagated from the uncertainties in the experimental intensities. The calculated values for $\mathrm{C}_{2} \mathrm{H}_{2}$ and $\mathrm{C}_{2} \mathrm{D}_{2}$ are in full agreement, both for the bond charge and the charge flux term. 
TABLE 10

Bond-charge parameter equations and resulting bond-charge parameters for $\mathrm{C}_{3} \mathrm{H}_{2}$ and $\mathrm{C}_{2} \mathrm{D}_{2}$

\begin{tabular}{|c|c|c|c|c|}
\hline & & & $\mathrm{C}_{2} \mathrm{H}_{2}$ & $\mathrm{C}_{2} \mathrm{D}_{2}$ \\
\hline \multirow{2}{*}{\multicolumn{5}{|c|}{$\begin{array}{c}\partial \mu_{z} / \partial S_{3}=-1.41421 q_{\mathrm{CH} / \mathrm{D}}-1.49977\left[\left(\partial q_{1} / \partial r_{1}\right)\right. \\
\left.-\left(\partial q_{3} / \partial r_{1}\right)-\gamma\left(\partial q_{2} / \partial r_{1}\right)\right] \\
\partial \mu_{x} / \partial S_{s x}=1.49977 q \mathrm{CH} / \mathrm{D}\end{array}$}} \\
\hline & & & & \\
\hline $\begin{array}{l}\mathrm{C}_{2} \mathrm{H}_{2} \\
\mathrm{C}_{2} \mathrm{D}_{2} \\
\text { Av. }\end{array}$ & $\begin{array}{l}0.992 \pm 0.020 \\
0.984 \pm 0.023 \\
0.988 \pm 0.030\end{array}$ & $\begin{array}{l}-0.0867 \pm 0.027 \\
-0.0917 \pm 0.024 \\
-0.0892 \pm 0.036\end{array}$ & & \\
\hline
\end{tabular}

${ }^{a}$ Units: $D \AA^{-1} \cdot{ }^{b}$ Units: $D \AA^{-2} \cdot{ }^{c} \gamma=r_{2} / r_{1}$.

It would be of interest to obtain individual values for the bond-charge derivatives, occurring in the charge flux term. Recently, we calculated ab initio values for these parameters [7]. In principle, by use of the ab initio ratios for these parameters, individual values could be calculated. However, the very small value of the charge flux term leads to very large uncertainties in the individual parameter values. Therefore no individual values are included in Table 10.

The dipole moment derivatives with respect to Cartesian displacement coordinates are easily obtained from the dmd's through

$\mathbf{P}_{\boldsymbol{X}}=\mathbf{P}_{S} \mathbf{B}+\mathbf{P}_{\rho} \beta$

where $\mathbf{B}$ denotes the transformation from Cartesian to symmetry coordinates, $\mathbf{S}=\mathbf{B X}, \beta$ is defined by $\rho=\beta \mathbf{X}$, the transformation form Cartesian to Eckart coordinates, and $\mathbf{P}_{\rho}$ is a $3 \times 6$ matrix containing the components of the molecular dipole moment. However, since acetylene has a zero permanent moment the last term of eqn. (10) vanishes. The matrix $\mathbf{P}_{\boldsymbol{X}}$ can be augmented in $N 3 \times 3$ submatrices, $N$ being the number of atoms of the molecule. These submatrices are the atomic polar tensors (apt's). In the case of acetylene the apt's obey the simple relationship

$\mathbf{P}_{X}^{\mathrm{H}_{1}}=\mathbf{P}_{X}^{\mathrm{H}_{2}}=-\mathbf{P}_{X^{i}}^{\mathrm{C}_{1}}=-\mathbf{P}_{X^{2}}^{\mathrm{C}_{2}}$

In Table 11 the averaged experimental apt's of acetylene are given, together with the corresponding apt's of propyne [21]. The $x x$ (or $y y$ ) elements of the $\mathbf{P}_{X}^{\mathrm{E}}$ apt's only depend on $\partial \mu_{x} / \partial S_{5 x}$ (or $\partial \mu_{y} / \partial S_{5 y}$ ), while the $z z$ element is completely determined by $\partial \mu_{z} / \partial S_{3}$. Therefore, the close correspondence between the $\partial \mu / \partial S$ parameters for acetylene and propyne (see Table 4 ) is also reflected in the corresponding elements of the $\mathbf{P}_{X}^{\mathrm{H}}$ apt's.

The difference between the corresponding elements of the $\mathbf{P}_{X}^{C}$ apt's for acetylene and propyne are much larger than for the $\mathbf{P}_{X}^{H}$ apt's. This has to be expected since the acetylene carbon polar tensor obeys the relationship $\mathbf{P}_{X}^{C}=$ 
TABLE 11

Experimental atomic polar tensors $\left(D A^{-1}\right)$ for acetylene and propyne

\begin{tabular}{lllllll}
\hline & Acetylene & \multicolumn{5}{c}{ Propyne $^{\mathrm{a}}$} \\
\hline $\mathbf{P}_{X}^{\mathrm{H}}$ & 0.988 & 0.0 & 0.0 & 1.013 & 0.0 & 0.0 \\
& 0.0 & 0.938 & 0.0 & 0.0 & 1.013 & 0.0 \\
& 0.0 & 0.0 & 0.894 & 0.0 & 0.0 & 0.871 \\
$\mathbf{P}_{X}^{C}$ & -0.988 & 0.0 & 0.0 & -1.207 & 0.0 & 0.0 \\
& 0.0 & -0.988 & 0.0 & 0.0 & -1.207 & 0.0 \\
& 0.0 & 0.0 & -0.894 & 0.0 & 0.0 & -2.101 \\
\hline
\end{tabular}

aThe values for the $x x$ and $y y$ elements of the propyne $P_{X}^{H}$ tensor as given in ref. 21 are in error. Here the correct $\nabla a l u e s$ are given.

$-\mathbf{P}$, this not being true for propyne. The $x x$ and $y y$ elements of the propyne $\mathbf{P}_{X}^{C}$ tensor contain not only a contribution of the $\mathrm{CCH}$ bending mode but also a contribution of the $\mathrm{CCC}$ bending mode, while the $z z$ element depends both on the $\equiv \mathrm{CH}$ strekching and the $\mathrm{C} \equiv \mathrm{C}$ stretching mode. It is clear from the foregoing discussion that the charge shifts in acetvlene due to small displacements of one of the hydrogen atoms are not very sensitive to substitution of the other hydrogen atom by a methyl group. However the same stubstitution already has a marked influence on the charge shirts that occur on displacements of the neighbouring carbon atom. In other words, the transferability of the acetylene $\mathbf{P}_{X}^{\mathrm{H}}$ tensor to propyne is very good, but the transferability of the apt of the neighbouring carbon atom is already rather poor. On the contrary, as mentioned before, both dmd values for acetylene show a very good transferability to propyne.

Therefore, from the viewpoint of transferability, the dmd parameters are more useful in this case. In general, still better transferability should be obtained by use of locally defined intensity parameters such as bcp's or eop's. Interesting work along these lines has already been performed by Zerbi, Gussoni and co-workers $[20,32-35]$. Similar work is now in progress in our laboratory.

\section{REFERENCES}

1 R. L. Kelly, R. Rollefson and B. S. Schurin, J. Chem. Phys., 19 (1951) 1595.

2 D. F. Eggers, I. C. Hisatsune and L. Van Alten, J. Phys. Chem., 59 (1955) 1124.

3 P. Varanasi and B. R. P. Bangaru, J. Quant. Spectrosc. Radiat. Transfer, 14 (1974) 839.

4 G. B. Mast and W. T. King, J. Phys. Chem., 80 (1976) 2004.

5 W. M. A. Smit; A.J. vain Straten and T. Visser, J. Mol. Struct., 48 (1978) 177.

6 K. Kim and W. T. King, J. Mol. Struct:, 57 (1979) 201 .

7 W. M.A. Smit and T. van Dam, J. Mol. Struct. Theochem, 88 (1982) 273.

8 F. B. Wilson, Jr. and A. I. Wells, J. Chem. Phys., 14 (1946) 578.

9 S. S. Penner and D. T. Weber, J. Chem. Phys., 19 (1951) 807.

10 J. D. Rogers, B. Rub, S. Goldman and W. B. Person, J. Phys. Chem., 85 (1981) 3727. 
11 K. F. Palmer, M. E. Mickelson and K. N. Rao, J. Mol. Spectrosc., 44 (1972) 131.

12 W. J. Lafferty and R. J. Thibault, J. Mol. Spectrosc., 14 (1964) 79.

13 A. G. Worthing and J. Geffner, Treatment of Experimental Data, Wiley, New York, 1947, Chap. XI.

14 T. A. Wiggins, E. K. Plyler and E. D. Tidwell, J. Opt. Soc. Am., 51 (1961) 1219.

15 J. F. Scott and K. N. Rao, J. Mol. Spectrose., 16 (1965) 15.

16 S. Ghersetti, J. Pliva and K. N. Rao, J. Mol. Spectrosc, 38 (1971) 53.

17 G. Strey and I. M. Mills, J. Mol. Spectrose., 59 (1976) 103.

18 B. L. Crawford, Jr., J. Chem. Phys., 20 (1952) 977.

19 W. M. A. Smit, J. Chem. Phys., 70 (1979) 5336.

20 P. Jona, M. Gussoni and G. Zerbi, J. Phys. Chem., 85 (1981) 2210.

21 J. H. G. Bode, W. M. A. Smit, T. Visser and H. D. Verkruijsse, J. Chem. Phys., 72 (1980) 6560 .

22 G. Jalsorszky and P. Pulay, J. Mol. Struct., 26 (1975) 277.

23 K. B. Wiberg and J. J. Wendoloski, J. Am. Chem. Soc., 100 (1978) 723.

$24 \mathrm{~K}$. B. Wiberg, private communication.

25 J. H. G. Bode and W. M. A. Smit, J. Phys. Chem., 84 (1980) 198.

26 S. J. Yao and J. Overend, Spectrochim. Acta, Part A, 32 (1976) 1059.

27 C. Domingo, R. Escribano, W. F. Murphy and S. Montero, J. Chem. Phys., 77 (1982) 4353 .

28 E. D. Tidwell and E. K. Plyler, J. Opt. Soc. Am., 52 (1962) 656.

29 R. M. Talley and A. H. Nielsen, J. Chem. Phys., 22 (1954) 2030.

30 A. J. van Straten and W. M. A. Smit, J. Mol. Spectrosc., 62 (1976) 297.

31 A. J. van Straten and W. M. A. Smit, J. Mol. Spectrose., 65 (1977) 202.

32 M. Gussoni, S. Abbate and G. Zerbi, J. Chem. Phys., 71 (1979) 3428.

33 P. Jona, M. Gussoni and G. Zerbi, J. Chem. Phys., 75 (1981) 1667.

34 M. Gussoni, S. Abbate, R. Sanvito and G. Zerbi, J. Mol. Struct., 75 (1981) 177.

35 P. Jona, M. Gussoni and G. Zerbi, J. Mol. Struct., 95 (1982) 15. 